Ces contradictions prennent une autre ampleur à la lecture de la conclusion, qui soumet trois orientations méthodologiques pour étudier une ville et en comprendre son évolution : réaliser une observation située à l'intérieur de la ville et à l'extérieur, étudier les liens étroits entre espaces et temps, considérer l'espace géographique comme un espace social. Ces principes finalement partiellement appliqués dans cet ouvrage - les habitants ont trop souvent été écartés; le temps, dont les dimensions sont complexes, est trop réduit à l'histoire; l'espace social a peu été abordé en tant qu'espace vécu, pratiqué et représenté - constituent malgré tout un agenda stimulant pour de nouvelles recherches urbaines sur des «villes du $\mathrm{XxI}^{\mathrm{e}}$ siècle ».

Février 2007, Matthieu Giroud

\title{
Roselyne de Villanova (dir.), Conjuguer la Ville. Architecture, Anthropologie, Pédagogie, Paris, L'Harmattan, 2007, 335 p., ISBN : 978-2-296-03889-9.
}

Derrière le titre suggestif du livre dirigé par Roselyne de Villanova, se cache un assemblage complexe de textes de nature assez variée. L'ouvrage résulte des travaux d'un atelier de recherche international. Son principal objectif est de questionner les synergies possibles entre «la vision des architectes et le regard des sciences sociales sur la ville et le logement» (p. 9). Il s'agit notamment de retracer l'histoire de disciplines qui ont contribué à l'élaboration de notions clefs pour l'analyse des formes et des espaces urbains, la réflexion se nouant de préférence autour d'expériences concrètes de collaboration entre sciences sociales et architecture.

Le sujet trouve un écho particulier dans le contexte portugais. Comme l'évoque brièvement Roselyne de Villanova dans l'introduction de l'ouvrage, la création en août 1974 du Serviço de Apoio Ambulatório (SAAL), pour résorber la crise du logement dans les villes portugaises, instaura un cadre original de coopération entre des associations d'habitants des quartiers dégradés, des équipes de techniciens (architectes, ingénieurs, sociologues) et les municipalités. Cette expérience s’inscrivait naturellement dans le nouvel ordre politique né de la Révolution des Eillets. Elle en partageait les valeurs et les objectifs, d'où sans doute son caractère utopique. Certes, elle ne marqua pas le début d'une collaboration durable : «les proximités sociopolitiques firent illusion sur l'existence d'une collaboration scientifique qui n'était pas vraiment l'enjeu» (p. 14). Les activités du SAAL, ses réalisations concrètes, les pratiques originales qu'elles ont générées tant en matière de gestion urbanistique que de gouvernance des villes, constituèrent pourtant de véritables laboratoires de politiques urbaines, reposant sur un dialogue constant entre revendications sociales et savoirs scientifiques et techniques. En fin de compte, ce sont aussi des formes originales de connaissance des sociétés urbaines qui émergèrent à cette occasion. Cette expérience éphémère n'a pas encore suffisamment attiré l'attention des sciences sociales et notamment des historiens. L'ouvrage dirigé par Roselyne de Villanova aurait pu être l'occasion de combler partiellement ce vide mais il faudra encore patienter ${ }^{1}$.

\footnotetext{
${ }^{1}$ À signaler cependant la récente publication d'une thèse de doctorat en architecture:
} 
Le livre est organisé en trois parties bien distinctes. Une première série de textes retrace le développement des recherches en anthropologie urbaine dans plusieurs pays (Italie, Mexique, Espagne, Portugal), l'exemple français étant traité à travers le cas particulier de l'anthropologie de l'espace. Une deuxième partie regroupe des récits d'expériences pédagogiques menées dans des écoles d'architecture européennes (Barcelone, Porto, Naples) et en Tunisie (Mahdia). Enfin, la troisième partie du livre apparaît comme la plus composite. Six chapitres discutent des outils d'analyse et des usages particuliers de l'espace ou des formes urbaines: les cités utopiques, le logement pavillonnaire, les espaces intermédiaires, la présence des employées migrantes dans l'espace domestique de familles napolitaines, l'exportation des projets d'architecture et la notion de topologie humaine.

L'ensemble peut dérouter et paraître assez hétéroclite. Le livre est cependant structuré autour d'une série de questionnements clairement énoncés dès l'introduction. Il propose d'abord une réflexion générale sur les formes de dialogue interdisciplinaire, confrontant les connaissances théoriques (sociologie, anthropologie) aux réflexions plus pratiques (architecture). Au-delà, il pose la question des collaborations possibles pour penser et construire ou planifier la ville. Tout l'ouvrage est parcouru par une réflexion originale sur l'application des sciences sociales, leur éventuelle influence dans la définition des politiques urbaines et les bénéfices que peuvent en tirer les praticiens (architectes ou urbanistes).

La première partie de l'ouvrage s'organise autour de la description de configurations particulières où l'histoire des disciples est influencée par des contextes sociaux et urbains spécifiques. À chaque fois, les anthropologues furent amenés à répondre à des défis posés par l'évolution des villes et des systèmes urbains de leur pays: un système urbain ancien et consolidé, en plein bouleversement à partir des années 1950, en Italie (Amalia Signorelli); une ville tentaculaire à Mexico (Angela Giglia); les contrastes régionaux en Espagne (Danielle Provansal); la référence dominante à la ruralité dans le cas du Portugal (Graça Indias Cordeiro). Comme le remarque Danielle Provansal, «en anthropologie des mondes contemporains, le choix des objets d'étude dépend largement de la demande sociale » (p. 66). Ce constat n'est pas neuf mais la confrontation entre différents cas nationaux amène ici des éclairages plus contrastés.

Dans la deuxième partie du livre, le point fort du propos sur l'enseignement des sciences sociales dans les écoles d'architecture est de privilégier une réflexion à partir d'expériences pratiques qui s'inscrivent elles aussi dans des histoires locales ou nationales. À partir du cas de la faculté d'architecture de Porto, Carolina Leite décrit comment un contexte spécifique d'exercice du métier d'architecte rend difficile l'introduction du discours critique des sciences sociales. L'originalité de ce texte, au sein de ce recueil, est de baser l'essentiel de sa réflexion sur la description d'une pratique professionnelle. La très forte médiatisation de quelques grands noms de l'architecture, qui évoluent dans un véritable «star-system », ne doit pas faire oublier la grande précarité professionnelle de la majorité des jeunes architectes. Pour ces derniers, la priorité n'est pas de penser de nouvelles pratiques mais plutôt de se détacher du groupe.

J.A. Bandeirinha, O processo SAAL e a Arquitectura no 25 de Abril de 1974, Coimbra, Imprensa da Universidade, 2007. 
Les derniers chapitres de l'ouvrage mériteraient des commentaires distincts. En effet, le fil conducteur du livre se ramifie peu à peu en une multitude d'interrogations relativement autonomes. Dans ce type d'ouvrage, il semble que l'on puisse difficilement faire l'impasse sur des études de cas précises qui viennent densifier et modérer une réflexion plus théorique. Pour revenir au thème central de l'ouvrage, les récits d'expériences originales ou la description d'usages particuliers enrichissent naturellement la réflexion sur les modes de penser voire de planifier la ville. Ainsi, en retraçant l'histoire de la réhabilitation du pavillonnaire dans la banlieue parisienne, Anne Debarre démontre bien comment cette « forme urbaine et architecturale du laissez-faire », autrefois vilipendée, a fini par s'imposer comme « modèle opposables à celui des ensembles groupés contemporains » (p. 220). Les enseignements à tirer de ces projets individuels sont multiples: "c'est dans la diversité de ses formes mais aussi de ses usages possibles que le pavillonnaire constitue une source de référence » (p. 228) pour les architectes contemporains. À travers un regard plus ethnographique, Adelina Miranda s'intéresse à la présence des employées à domicile issues de l'immigration des pays de l'Est de l'Europe, dans l'espace domestique de familles napolitaines. Cette étude permet de questionner les «valeurs de la proximité et de la distance socioculturelle " (p. 251) à partir d'une situation inédite de cohabitation entre les migrantes et les familles. Les employées travaillent, et parfois vivent, dans des lieux habités qui ne sont pas conçus pour les accueillir. Adelina Miranda s'interroge sur ce «processus d'adéquation » à l'origine de «formes de négociation socioculturelle spécifiques » (p. 258). D'autres textes adoptent des approches plus théoriques. Le propos peut alors devenir allusif et parfois déroutant. Quand Jean-Michel Léger parle de "première internationalisation de l'architecture au bon vieux temps des modernes (de Aalto à Le Corbusier)» (p. 261), on comprend qu'il fait allusion à la médiatisation des réalisations, notamment à travers la diffusion de la photographie. La formulation apparaît cependant un peu rapide.

Toutefois, au-delà de la diversité des usages et des objets analysés, un nombre limité de thèmes s'impose par leur importance et leur transversalité. Ainsi, l'idée de «métissage » se retrouve au cœur de la réflexion sur les espaces intermédiaires comme forme architecturale et comme métaphore sociale (Roselyne de Villanova) ou sur l'exportation des projets d'architecture dans le domaine du logement (JeanMichel Léger). Il s'agit d'une notion consubstantielle aux sociétés urbaines mais aussi aux créations et fabrications de formes qui se réactualisent et se renouvellent constamment. Cette idée se retrouve aussi bien dans les études anthropologiques que dans les pratiques des architectes. Elle permet donc de relativiser l'opposition entre sciences appliquées et sciences fondamentales. Comme le remarque Daniel Pinson dans la postface de l'ouvrage, l'architecture produit aussi une connaissance sur la ville.

Ce livre s'avère donc particulièrement riche. Cependant, bien qu'il se veuille ouvert et clairement pluridisciplinaire, il n'est pas d'un abord facile pour les lecteurs peu familiarisés avec les débats internes à l'anthropologie. Par ailleurs, l'édition n'a pas toujours été très soignée: plusieurs références citées dans les textes sont absentes des bibliographies de fin de chapitre. Enfin, on pourra trouver quelques points discutables et ressentir des regrets. Au cœur de ce projet se trouve un constat sur la circulation des textes de référence et des modèles. On aimerait en 
savoir plus sur le fonctionnement de ces réseaux, le rôle des institutions (écoles d'architecture, universités, pouvoirs politiques) et des différents acteurs. Quelle est la dynamique de cette configuration culturelle instable constituée par les pays de langues latines représentés dans ce recueil? Quelles relations s'établissent entre pratiques ou contextes locaux et les modèles transnationaux? On peut enfin signaler une autre faille d'importance. On trouvera bien peu de références au rôle des politiques ou des administrateurs, telles les municipalités. Il semble difficile de penser les relations entre architecture et sciences sociales sans tenir compte de l'évolution des formes de professionnalisation des métiers de la ville et de l'urbain. La définition des compétences et des savoirs sur la ville correspond aussi à un processus qui court tout au long du $\mathrm{XX}^{\mathrm{e}}$ siècle, en fonction de l'évolution et des différentes déclinaisons ou mises en pratique des projets réformateurs.

Mars 2007, Frédéric Vidal 